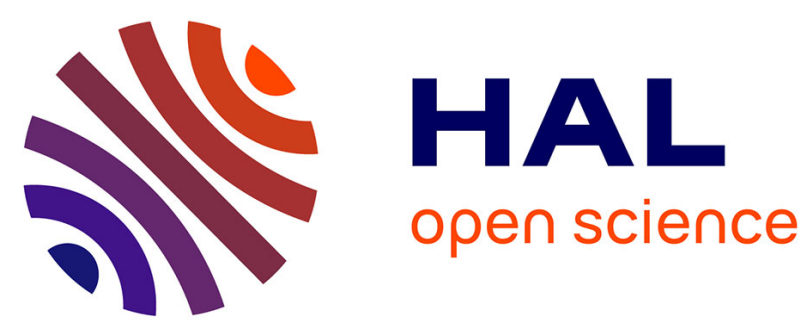

\title{
UTILISATION DE L'INDICE RASTI ET DU TEMPS DE RÉVERBÉRATION INITIAL POUR L'ANALYSE DES PROBLÈMES D'ACOUSTIQUE ARCHITECTURALE
}

\author{
J. Migneron
}

\section{To cite this version:}

J. Migneron. UTILISATION DE L'INDICE RASTI ET DU TEMPS DE RÉVERBÉRATION INITIAL POUR L'ANALYSE DES PROBLÈMES D'ACOUSTIQUE ARCHITECTURALE. Journal de Physique IV Proceedings, 1992, 02 (C1), pp.C1-135-C1-138. 10.1051/jp4:1992126 . jpa-00251197

\author{
HAL Id: jpa-00251197 \\ https://hal.science/jpa-00251197
}

Submitted on 1 Jan 1992

HAL is a multi-disciplinary open access archive for the deposit and dissemination of scientific research documents, whether they are published or not. The documents may come from teaching and research institutions in France or abroad, or from public or private research centers.
L'archive ouverte pluridisciplinaire HAL, est destinée au dépôt et à la diffusion de documents scientifiques de niveau recherche, publiés ou non, émanant des établissements d'enseignement et de recherche français ou étrangers, des laboratoires publics ou privés. 


\title{
UTILISATION DE LINDICE RASTI ET DU TEMPS DE REVERBERATION INITIAL POUR LANALYSE DES PROBLÈMES D'ACOUSTIQUE ARCHITECTURALE
}

\author{
J.G. MIGNERON \\ Laboratoire d'Acoustique de l'Université Laval, CRAD 1636 Pavillon Félix-Antoine Savard, Cité \\ Universitaire, Québec GIK 7P4, Canada
}

\begin{abstract}
Correlation between the Rasti index and the impulse response, namely the EDT-5,EDT-10 and EDT-15 dB, has been analysed, both in laboratory and during experiments conducted in multi-purpose halls and in large amphitheatres equipped with sound reinforcement systems. The close relationship between the RASTI index and the EDT measurements will be demonstrated. We will also show the necessity to measure the EDT's at the same localization and with the same directivity as the normal sources of the hall; for exemple, with the use of a powerful omnidirectional source in order to study the acoustical behavior of orchestra shell (Louis Fréchette Hall of the Grand theâtre de Québec or Albert Rousseau Hall) or with the measurement of impulse response through the sound system of a large amphitheatre (Montreal Olympic Stadium). In addition to provide a better understanding of the parameters governing the intelligibility, the proposed approach could be very helpful to the acoustical design of new and to be renovated halls (New Richmond Theatre, The National Theater Institute of Montreal).
\end{abstract}

\section{INTRODUCTION}

Le STI et la procédure condensée du RASTI constituent une même méthode objective de mesure de la qualité de transmission de la parole qui quantifie particulièrement bien l'intelligibilité; il s'agit de la méthode mise au point par HOUTGAST et STEENEKEN en 1980 [1,2]. Les deux paramètres susceptibles d'influencer le STI ("Speech Transmission Index") ou l'indice simplifié RASTI ("Rapid Speech Transmission Index") sont le niveau du bruit de fond et le temps de réverbération; dans un local réverbérant, ces deux paramètres affectent la dynamique du signal perçu, tout particulièrement au tout début de chacune des décroissances de l'énergie acoustique. Pour l'obtention de l'indice RASTI, le signal de test utilisé consiste en deux octaves de bruit rose, centrés sur les bandes de $500 \mathrm{~Hz}$ et $2 \mathrm{KHz}$; les niveaux, dans ces bandes d'octave, étant choisis pour être représentatifs du niveau moyen de la voix (sonorisée ou non). Les modulations de basses fréquences présentes dans la voix humaine sont simulées par neuf fréquences discrètes de modulation. Les mesures RASTI consistent en une analyse du signal résultant à la position d'écoute, de façon à calculer le facteur de réduction du niveau de modulation pour chacune de ces neuf fréquences de modulation. Si le rapport signal sur bruit devient prédominant la fonction de transfert de modulation MTF donne une réponse plate, c'est-à-dire que toutes les fréquences de modulation sont affectées de la même manière; par contre, si c'est le temps de réverbération qui domine, la fonction de transfert de modulation présente une pente négative, parce que la réverbération affecte les fréquences de modulations les plus hautes.

Il a été démontré, également, une cohérence évidente avec d'autres méthodes consacrées aux salles de concert comme le "Clarity Index" ( $\mathrm{C}_{80}$, de REICHARDT, 1981), indice qui peut être complété par la modélisation de l'intelligibilité de BRADLEY $[3,4]$. Néanmoins, le paramètre de la réverbération initiale EDT apparaît plus facile à obtenir et à modéliser dans le contexte d'une réverbération variable, à la fois temporellement, au long de la décroissance, et spatialement (ce qui est généralement le cas dans un grand local très réverbérant), puisque ce paramètre ne s'attache pas à l'énergie tardive.

\section{RELATION ENTRE L'INDICE RASTI ET LE TEMPS DE RÉVERBÉRATION INITIAL}

L'utilisation de l'indice RASTI et de la réponse impulsionnelle, notamment la détermination exacte et précise des indices EDT -5, EDT -10 et EDT -15 dB a été valorisée ces dernières années, tant en laboratoire qu'au cours de différentes expertises dans plusieurs salles de spectacle, dédiées ou non à la musique, ou dans de grands locaux sonorisés (à l'aide des nouveaux analyseurs rapides et d'un échantillonnage au tiers d'octave, au 1/100 sec.ou plus serré). On notera, à ce sujet, que les logiciels d'exploitation des analyseurs doivent être modifiés pour obtenir ces trois indices de façon répétitive, à partir d'impulsions variables. 
Afin d'éclairer cette relation de dépendance entre l'intelligibilité et le EDT, il avait été fait état au congrès de Lyon [5,7] d'une expérience menée dans un local vide de plus de $50 \mathrm{~m}$ de long, avec une répartition homogène de l'absorption et de la diffusion; cette expérience ayant consisté à mesurer simultanément l'indice RASTI et le temps de réverbération initial EDT, à $-5,-10$ et $-15 \mathrm{~dB}$, pour les bandes d'octave de 500 et $2000 \mathrm{~Hz}$, la source impulsive utilisée ayant une directivité peu prononcée. Après avoir calculé les valeurs des temps de réverbération initiaux EDT susceptibles de redonner l'indice RASTI obtenu (à partir des fonctions de transfert de modulation réellement mesurées), il avait été vérifié que la régression la plus proche, dans les deux bandes de fréquence, était bien EDT $-5 \mathrm{~dB}$. Suite à ces résultats, il apparaît donc nécessaire de mesurer la toute première décroissance de l'énergie, pour pouvoir modéliser convenablement l'indice RASTI et l'intelligibilité.

\section{Différents eXEMPleS de SAlLes de SPECTACle OU DE CONCERT}

Comme le montre, à titre d'exemple, le Tableau ${ }^{\circledR} 1$, relevé dans la salle de l'École Nationale de Théâtre à Montréal avant sa rénovation (une des plus vieilles salles de spectacle du Canada), cette étroite relation, entre l'indice d'intelligibilité RASTI obtenu à partir des pertes de modulation et les valeurs réellement mesurées du EDT, a été documentée et mise en évidence, dans différentes expertises d'analyse acoustique de salles de spectacle ou de concert (voir également la Figure $\mathrm{n}^{2} 1$ ).

TABLEAU N¹:Mesure des temps de réverbération initiaux EDT $-5,-10$ et $-15 \mathrm{~dB}$ et de l'indice RASTI dans un théatre ancien (École Nationale de Théatre de Montréal, avant rénovation).

\begin{tabular}{|c|c|c|c|c|c|c|c|c|c|c|c|c|}
\hline Localisation & RASTI & \multicolumn{3}{|c|}{ EDT $500 \mathrm{~Hz}$} & RASTI & \multicolumn{3}{|c|}{ EDT $1000 \mathrm{~Hz}$} & \multicolumn{3}{|c|}{ EDT $2000 \mathrm{~Hz}$} & RASTI \\
\hline Parterre & $\%$ & $-5 \mathrm{~dB}$ & $-10 \mathrm{~dB}$ & $-15 \mathrm{~dB}$ & $\%$ & $-5 \mathrm{~dB}$ & $-10 \mathrm{~dB}$ & $-15 \mathrm{~dB}$ & $-5 \mathrm{~dB}$ & $-10 \mathrm{~dB}$ & $-15 \mathrm{~dB}$ & $\%$ \\
\hline Troisième rangóe & 63 & 0.28 & 1.17 & 1.50 & 61 & 0.38 & 1.50 & \begin{tabular}{|l}
1.63 \\
\end{tabular} & 0.24 & 0.94 & 1.28 & 65 \\
\hline Centre parterre & 53 & 0.44 & 1.91 & 1.99 & 46 & 0.75 & 1.67 & 2.17 & 0.56 & 1.08 & 1.38 & 58 \\
\hline Fond du parterne & 54 & 1.12 & 1.41 & 1.88 & 42 & 0.94 & 1.59 & 1.66 & 0.47 & 0.80 & 1.25 & 65 \\
\hline Balcon & 48 & 1.31 & 1.56 & 1.66 & 44 & 0.23 & 0.33 & 1.33 & 0.56 & 1.41 & 1.62 & 52 \\
\hline
\end{tabular}

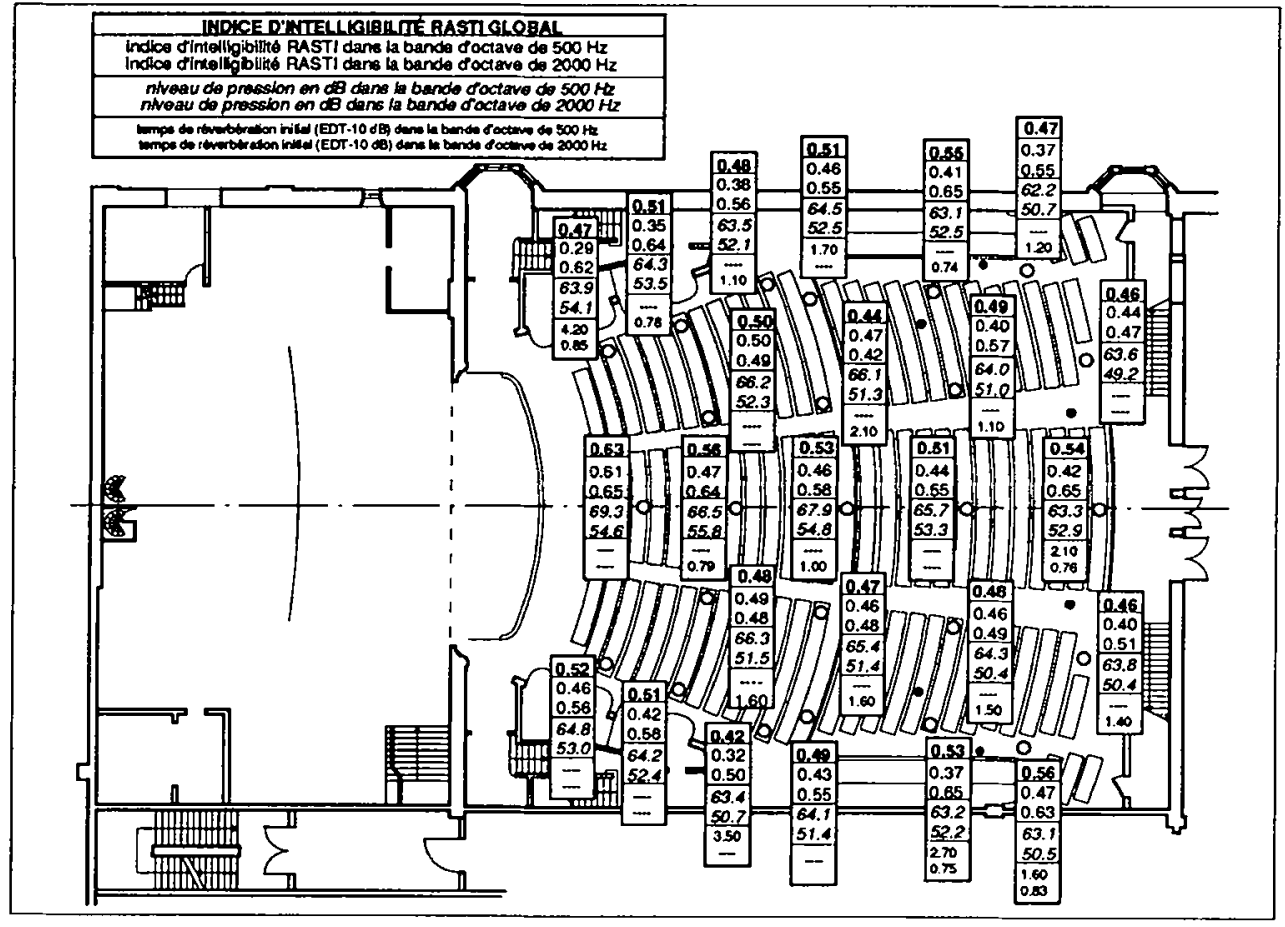

FIGURE NN1: Mesure de l'indice RASTI dans un théâtre ancien, avec indication du EDT estimé da partir des mesures d'intelligibilité (École Nationale de Théatre de Montréal, avant rénovation). 
En fait, la lecture simultanée des trois indices EDT -5, -10 et -15 dB apporte souvent une dimension supplémentaire à la compréhension du comportement acoustique local (même si les autres indices ne sont pas significatifs).

La nécessité d'acquérir les temps de réverbération initiaux à partir des mêmes localisations que les sources ordinaires des salles de spectacle analysées et avec la même directivité que ces sources a été également mise en évidence. D'où l'emploi, par exemple, d'une source omnidirectionnelle de forte puissance, pour l'étude du comportement des conques d'orchestre. Le Tableau $\mathrm{n}^{2} 2$ montre, à ce sujet, l'excellente relation obtenue, avec ce genre de source, dans la conque d'orchestre de la salle Louis Fréchette du Grand-Thêâtre de Québec, entre les indices EDT, RASTI et C 80 . De même, on montrera comment les mesures RASTI ont permis un meilleur réglage de la conque d'orchestre de la salle Albert Rousseau.

TABLEAU N22: Principaux résultats liés d la réponse impulsionnelle d'une salle de concert (salle Louis Fréchette du Grand-Théâtre de Québec).

\begin{tabular}{|c|c|c|c|c|c|c|c|c|c|c|c|c|c|c|c|c|c|c|}
\hline \multirow{2}{*}{$\begin{array}{l}\text { Localisation } \\
\text { Bandes d'oct. }\end{array}$} & \multicolumn{3}{|c|}{$\begin{array}{l}\text { Temps de réverb. } \\
\text { RT } 60 \text { en sec. }\end{array}$} & \multicolumn{3}{|c|}{$\begin{array}{c}\text { Réverb. initiale } \\
\text { EDT-5 } \mathrm{dB}\end{array}$} & \multicolumn{3}{|c|}{$\begin{array}{l}\text { Réverb. initiale } \\
\text { EDT-10 dB }\end{array}$} & \multicolumn{3}{|c|}{$\begin{array}{l}\text { Réverb. initiale } \\
\text { EDT-15 dB }\end{array}$} & \multicolumn{3}{|c|}{$\begin{array}{l}\text { Indice de clarté } \\
\text { C } 80 \mathrm{~ms}\end{array}$} & \multicolumn{3}{|c|}{$\underset{\%}{\text { Indice RASTI }}$} \\
\hline & 500 & $1 \mathbf{k}$ & $2 k$ & 500 & $1 \mathrm{k}$ & $2 k$ & 500 & $1 \mathrm{k}$ & $2 \mathbf{k}$ & 500 & $1 \mathbf{k}$ & $2 k$ & 500 & $1 \mathbf{k}$ & $2 k$ & 500 & $2 \mathrm{k}$ & Tot. \\
\hline Avant-scène & 1.58 & 1.57 & 1.46 & 0.22 & 0.45 & 0.62 & .73 & 0.78 & 0.75 & 0.94 & 1.14 & 1.32 & 0.86 & 0.92 & 0.76 & 80 & 71 & 75 \\
\hline Parteme G1 & $\frac{1.85}{1.85}$ & 1.72 & 1.50 & 0.15 & 0.85 & $\frac{0.12}{0.12}$ & .46 & \begin{tabular}{|l|}
0.10 \\
0.71
\end{tabular} & 0.73 & 0.88 & 1.13 & 1.07 & 0.75 & 0.67 & 0.74 & 46 & 56 & 53 \\
\hline Parterte T1 & 1.90 & 1.84 & 1.54 & 0.10 & 1.85 & 1.10 & .81 & 1.23 & 1.08 & 1.28 & 1.34 & 1.09 & 0.73 & 0.77 & 0.68 & $\begin{array}{l}49 \\
(74)\end{array}$ & $\begin{array}{c}49 \\
(77) \\
\end{array}$ & $\begin{array}{l}49 \\
(76)\end{array}$ \\
\hline Balcon $\mathrm{H} 1$ & 1.86 & 1.66 & 1.57 & .47 & 0.18 & 290 & 0.82 & 0.90 & 0.58 & 0.98 & 0.93 & 0.86 & 0.74 & $\overline{0.79}$ & 0.77 & 49 & 55 & 52 \\
\hline
\end{tabular}

Remarque: les valeurs entre parenthèses ont été obtenues avec le système de sonorisation de la salle.

Quant aux systèmes de sonorisation dans les grands locaux, il convient, préférablement, de procéder à la mesure de la réponse impulsionnelle au travers même du système de haut-parleurs (voir Tableau nọ, à propos du nouveau système de sonorisation du Stade Olympique de Montréal). En plus de mieux comprendre les paramètres responsables de l'intelligibilité, les approches proposées peuvent aider grandement dans le design des nouvelles salles ou la rénovation des salles de spectacle plus anciennes. À titre d'exemple, le Tableau $n^{93}$ montre ce qu'il est possible d'obtenir avec une bonne conception acoustique (nouvel auditorium de New-Richmond, 900 places), l'indice de RASTI global restant toujours supérieur à $65 \%$ sans sonorisation.

TABLEAU N23: Principaux résultats des mesures RASTI dans un nouvel auditorium, avec et sans sonorisation (Théatre de New-Richmond).

\begin{tabular}{|c|c|c|c|c|c|c|c|c|c|c|}
\hline Localisation & \multicolumn{3}{|c|}{$\begin{array}{l}\text { RASTI mesuré en \% } \\
\text { (sans sonorisation) }\end{array}$} & \multicolumn{3}{|c|}{$\begin{array}{c}\text { RASTI mesuré en \% } \\
\text { (avec syst. de haut-parleurs) }\end{array}$} & \multicolumn{2}{|c|}{$\begin{array}{c}\text { EDT détuit des } \\
\text { mesures RASTI } \\
\text { sans sonorisation }\end{array}$} & \multicolumn{2}{|c|}{$\begin{array}{l}\text { EDT déduit des } \\
\text { mesures RASTI } \\
\text { avec sonorisation }\end{array}$} \\
\hline Bandes d'octave $\mathrm{Hz}$ & 500 & $2 k$ & Total & 500 & $2 \mathbf{k}$ & Total & 500 & $2 k$ & 500 & $2 k$ \\
\hline Troisième rangée du parterre & 63 & 88 & 77 & 59 & 82 & 72 & .80 & .20 & .97 & .29 \\
\hline Dixième rangé du parterre & 60 & 73 & 67 & 69 & 82 & 76 & .91 & .50 & .64 & .30 \\
\hline Quinzième rangée du parterre & 63 & 75 & 70 & 71 & 76 & 74 & .81 & .44 & .58 & .32 \\
\hline Fond du parterre & 65 & $\overline{84}$ & 76 & 78 & 92 & 86 & .76 & .26 & 70 & .15 \\
\hline
\end{tabular}

\section{Simulation DE L'INTELLIGIBILITÉ ET SYSTEMES DE SONORISATION}

L'influence de la directivité de la source sur les temps de réverbération initiaux a été clairement mise en évidence: une grande directivité entraîne nécessairement une part de champ diffus plus importante, pour les points qui ne sont pas dans le champ direct de la source et, réciproquement, le niveau relatif du son direct est plus élevé pour les points directement exposés.

La recherche a démontré que pour mettre au point une procédure de modélisation convenable de l'intelligibilité pour les nouveaux systèmes de sonorisation dans des locaux fortement réverbérants, il faut idéalement disposer du temps de réverbération initial EDT, avec une localisation exacte du point source et une directivité similaire à celles des futurs haut-parleurs. Cependant, la modélisation de l'intelligibilité peut rester significative, avec une connaissance plus fragmentaire du champ réverbéré (puisque le véritable EDT local ne peut être directement mesuré sans les futurs haut-parleurs); il est également possible d'envisager l'usage d'un système de haut-parleurs de référence, durant la mesure. On peut toujours calculer l'intelligibilité à partir d'une connaissance spatialement limitée du EDT $-5 \mathrm{~dB}$, soit en interpolant les valeurs les plus proches de la source et en tenant compte de sa directivité. 
À titre d'exemple, lors de tests préliminaires sur le rendement des nouveaux haut-parleurs du Stade Olympique de Montréal [6], on a procéde, en plus des mesures de pression et de RASTI, à une analyse de la réponse impulsionnelle à travers le système sonorisation, soit avec la localisation et la directivité quasidéfinitives des nouveaux haut-parleurs. L'introduction, dans le modele informatique, des valeurs mesurées de EDT - 5dB a permis de simuler, avec une grande précision cette fois, les valeurs de l'indice RASTI escomptées.

TABLEAU N24:Modélisation de l'intelligibillité d travers un système de sonorisation (nouveau système de sonorisation du Stade Olympique de Montréal).

\begin{tabular}{|c|c|c|c|c|c|c|c|c|c|c|}
\hline Localisation & \multicolumn{2}{|c|}{$\begin{array}{c}\text { EDT déduit des } \\
\text { mesures de réverb. }\end{array}$} & \multicolumn{2}{|c|}{$\begin{array}{l}\text { EDT -5dB mesuré } \\
\text { avec haut-parleurs }\end{array}$} & \multicolumn{3}{c|}{ RASTI modélisé } & \multicolumn{2}{c|}{ RASTI mesuré réellement } \\
\hline Bandes d'octaves Hz & 500 & $2 \mathrm{k}$ & 500 & $2 \mathrm{k}$ & 500 & $2 \mathrm{k}$ & Total & 500 & $2 \mathrm{k}$ & Total \\
\hline Bas de la section 100 & 3.43 & 2.53 & 0.70 & 0.36 & 62 & 76 & 73 & 74 & 66 & 70 \\
\hline Axe haut-parleurs section 100 & 3.28 & 3.04 & 0.65 & 0.39 & 68 & 78 & 70 & 72 & 70 & 71 \\
\hline Axe haut-parleurs sect. 2-300 & 2.15 & 2.05 & 0.51 & 0.39 & 73 & 80 & 76 & 77 & 73 & 75 \\
\hline Haut de la section 300 & 2.79 & 1.22 & 0.59 & 0.62 & 69 & 71 & 69 & 71 & 70 & 71 \\
\hline
\end{tabular}

Ces résultats sont fort encourageants, puisqu'ils laissent apparaître à la fois la complexité du problème et une solution possible à la modélisation exacte de l'intelligibilité.

\section{Conclusion}

Dans une salle existante, que ce soit pour l'intelligibilité de la voix proprement dite ou pour la qualité de l'écoute en général, fusse-t-elle musicale, l'emploi des indices RASTI et EDT $-5,-10$ et $-15 \mathrm{~dB}$ permet d'éclairer, de façon complémentaire et analytique, le comportement acoustique, qu'il s'agisse d'une salle de spectacle ou de n'importe quel grand local sonorisé. Ces indices peuvent être mesurés seuls ou bien coordonnés, avec la réponse impulsionnelle conventionnelle et la détermination d'un indice tel que $\mathrm{C}_{80}$ (dans une salle symphonique notamment). Pour cerner complètement les résultats obtenus, il faut s'assurer que tous ces paramètres soient bien mesurés, avec un processus de mesure précis et répétitif, à partir d'un point source représentatif d'un usage bien déterminé de la salle et avec une directivité similaire à celle des sources réellement en présence, lors d'un spectacle ou d'un concert.

Au plan prédictif, la détermination du EDT (à l'aide d'un modèle de tir de rayons par exemple) pourra permettre de déduire avec une précision suffisante l'intelligibilité escomptée. Enfin, en matière de sonorisation, pour les grands locaux très réverbérants, la simulation exacte du STI ou de l'indice RASTI exigera, non seulement une connaissance précise de la réverbération locale au point d'écoute, mais, également, celle de l'influence de la localisation et de la directivité des haut-parleurs sur la réponse impulsionnelle résultante.

\section{RÉFÉRENCES}

[1] Houtgast, T., SteEneken, H.J.M. et PlOMP, R.: "Predicting speech intelligibility in rooms from the modulation transfer function in room - 1. General room acoustics", pp. 60-72, in Acustica, vol.46, 1980.

[2] HOUTGAST, T., STEENEKEN, H.J.M.: "A review of the MTF concept in room acoustics and its use for estimating speech intelligibility in auditoria", pp.-1069-1077, in Journ. of Acoust. Soc. Am., vol. $77,1985$.

[3] BRADLEY, J.S.: "Predictors of speech intellligibility in rooms", pp. 837-845, in Journ. of Acoust. Soc. Am.,vol.80, $\mathrm{n}^{2} 3,1986$.

[4] BRADLEY, J.S. et HALLIWELL, R.E.: "Making auditorium acoustics more quantitative", pp. 16-23, in Sound \& Vibr. vol.23, $n^{2} 2,1989$.

[5] Migneron, J.-G. et LECLERC, D.: "Modélisation de l'intelligibilité pour le nouveau sytème de sonorisation du Stade Olympique de Montréal", pp. 39-45, in Proceedings, Semaine canadienne d'acoustique, Halifax, octobre 1989.

[6] Migneron, J.-G.: "Modélisation de l'intelligibilité dans les locaux réverbérants", pp. 49-55, in Proceedings, Semaine canadienne d'acoustique, Montréal, octobre 1990.

[7] MIGNERON, J.-G. et LECLERC, D.: "Modélisation de l'intelligibilité et sonorisation du Stade Olympique de Montrél", pp.1093-1096, in Colloque de physique , tome 51, février 1990.

[8] MIGNERON, J.-G.: "Analyse acoustique du Stade Olympique de Montréal", 16 p. in Proceedings, Semaine canadienne d'acoustique, Toronto, octobre 1988. 